PROCEEDINGS OF THE

AMERICAN MATHEMATICAL SOCIETY

Volume 133, Number 9, Pages 2507-2513

S 0002-9939(05)08093-7

Article electronically published on March 31, 2005

\title{
THE WEAK DIMENSIONS OF GAUSSIAN RINGS
}

\author{
SARAH GLAZ \\ (Communicated by Bernd Ulrich) \\ Dedicated to Wolmer Vasconcelos
}

\begin{abstract}
We provide necessary and sufficient conditions for a Gaussian ring $R$ to be semihereditary, or more generally, of $w \cdot \operatorname{dim} R \leq 1$. Investigating the weak global dimension of a Gaussian coherent ring $R$, we show that the only values $w \cdot \operatorname{dim} R$ may take are 0,1 and $\infty$; but that $f P \cdot \operatorname{dim} R$ is always at most one. In particular, we conclude that a Gaussian coherent ring $R$ is either Von Neumann regular, or semihereditary, or non-regular of $w \cdot \operatorname{dim} R=\infty$.
\end{abstract}

\section{INTRODUCTION}

Let $R$ be a commutative ring, and let $x$ be an indeterminate over $R$. For a polynomial $f \in R[x]$, denote by $c(f)$ - the content of $f$-the ideal of $R$ generated by the coefficients of $f$. For two polynomials $f$ and $g$ in $R[x]$,

$$
c(f g) \subseteq c(f) c(g) .
$$

A polynomial $f$ is called a Gaussian polynomial if this containment becomes equality for every polynomial $g$ in $R[x]$. A $\operatorname{ring} R$ is called a Gaussian ring if every polynomial with coefficients in $R$ is a Gaussian polynomial. Both definitions first appeared in Tsang's thesis 12, where it is also proved that polynomials with invertible, or more generally locally principal, content ideals are Gaussian polynomials. The question whether the converse holds received a great deal of attention in recent years; Glaz \& Vasconcelos [6, 7], Heinzer \& Huneke [8, Loper \& Roitman [9], and Lucas [10]. 3] provides a survey of the results obtained until the year 2000, and an extensive reference list.

The present article is concerned with a related, but different, aspect of this topic, namely the characterization of Gaussian rings. Tsang [12] and Gilmer [4] provided a very elegant homological characterization of Gaussian domains. Recall that a domain $R$ is a Prüfer domain if every finitely generated ideal of $R$ is invertible. The result of Tsang, and Gilmer, (recently given a new proof by the work of Loper \& Roitman) stated that a domain $R$ is Gaussian if and only if it is a Prüfer domain. Our concern is with the form this characterization takes when the ring is not a domain.

A homological generalization to rings $R$ of the Prüfer property for domains, is the condition: $R$ has weak global dimension less or equal to one, $w$. $\operatorname{dim} R \leq 1$. Recall

Received by the editors February 8, 2004.

2000 Mathematics Subject Classification. Primary 13F05, 13D05.

Key words and phrases. Gaussian rings, semihereditary rings, weak dimension.

(C)2005 American Mathematical Society Reverts to public domain 28 years from publication 
that this condition is equivalent to asking that all ideals of $R$ be flat. A subfamily of this class of rings is the class of semihereditary rings. Recall that a ring is semihereditary if every finitely generated ideal of $R$ is projective. Semihereditary rings $R$ are precisely those rings $R$ with $w \cdot \operatorname{dim} R \leq 1$ which are coherent. Domains of weak global dimension less or equal to 1 , in particular, semihereditary domains, are Prüfer domains. And in Section 2 we show that rings of $w \cdot \operatorname{dim} R \leq 1$ are Gaussian rings. We also provide a number of necessary and sufficient conditions for a Gaussian ring to be semihereditary, or more generally, of weak global dimension less or equal to one.

In Section 3 we consider the question: For a Gaussian ring $R$, what values may $w . \operatorname{dim} R$ take? We provide a complete answer to this question in case the ring $R$ is coherent. In particular, we prove that a Gaussian coherent ring $R$ always satisfies $f P \cdot \operatorname{dim} R \leq 1$, and is either regular, in which case $w \cdot \operatorname{dim} R \leq 1$ (that is, $R$ is either semihereditary or Von Neumann regular); or is not regular and w.dim $R=$ $\infty$. We conclude, by providing examples that show that both $f P \cdot \operatorname{dim} R=0$ and $f P \cdot \operatorname{dim} R=1$ are realizable in Gaussian, Noetherian rings of $w \cdot \operatorname{dim} R=\infty$.

Tsang [12], D.D. Anderson [1, and D.D. Anderson \& V. Camillo [2] considered various aspects of Gaussian rings, touching indirectly on some of the topics of this article.

The term local ring means a not necessarily Noetherian ring with only one maximal ideal.

\section{WEAK GLOBAL DIMENSION ONE}

There are two conditions that one can impose on the set of all principal ideals of a ring $R$, in order to obtain some control on the behavior of the zero divisors of $R$. A ring $R$ is called a PF ring if all principal ideals of $R$ are flat. The PF condition on $R$ is equivalent to $R$ being locally a domain [5] Theorem 4.2.2]. A ring $R$ is called a PP ring or a Weak Baer ring, if all principal ideals of $R$ are projective. The $\mathrm{PP}$ condition, stronger than the $\mathrm{PF}$ condition, is equivalent to $R$ being locally a domain, and $Q(R)$, the total ring of quotients of $R$, being Von Neumann regular [5, Theorem 4.2.10].

The following lemma appears in Tsang's thesis [12], but was never published. Because the result is both interesting and useful, we give a proof below. The proof is somewhat different, and much shorter, than Tsang's proof.

Lemma 2.1. Let $R$ be a local Gaussian ring, and let $I=\left(a_{1}, \ldots, a_{n}\right)$ be a finitely generated ideal of $R$. Then $I^{2}=\left(a_{i}^{2}\right)$, for some $1 \leq i \leq n$.

Proof. We first assume that $I$ is two generated, $I=(a, b)$. Let $f(x)=a x+b$, and consider $g(x)=a x-b$. Then $c(f g)=c(f) c(g)$, implies that

$$
(a, b)^{2}=\left(a^{2}, b^{2}\right) .
$$

Let $h(x)=b x+a$. Then $c(f h)=c(f) c(h)$, implies that

$$
(a, b)^{2}=\left(a b, a^{2}+b^{2}\right) .
$$

It follows that $\left(a^{2}, b^{2}\right)=\left(a b, a^{2}+b^{2}\right)$, and we can write $a^{2}=r a b+s\left(a^{2}+b^{2}\right)$ for some $r$ and $s$ in $R$. Rewrite this equality as

$$
(1-s) a^{2}+r a b+s b^{2}=0 .
$$


Since $R$ is a local ring, either $s$ or $1-s$ is a unit in $R$. Assume that $1-s$ is a unit in $R$, and $a^{2}+\alpha a b+\beta b^{2}=0$, for $\alpha=r / 1-s$ and $\beta=s / 1-s$. It follows from this equality, that if we can show that $a b \epsilon\left(b^{2}\right)$, then $(a, b)^{2}=\left(b^{2}\right)$ as desired. Let $k(x)=(a+\alpha b) x-b$. Then $c(f k)=c(f) c(k)$, implies that

$$
\left(a(a+\alpha b),-a b+b(a+\alpha b),-b^{2}\right)=\left(b^{2}\right)=(a, b)((a+\alpha b), b) .
$$

In particular, $a b \epsilon\left(b^{2}\right)$.

Note that the above argument implies that for any two elements $a$ and $b, a b \epsilon\left(b^{2}\right)$ or $\left(a^{2}\right)$. It follows that $I^{2}=\left(a_{1}, \ldots, a_{n}\right)^{2}=\left(a_{1}^{2}, \ldots, a_{n}^{2}\right)$ for all $n$. An induction on $n$ yields $I^{2}=\left(a_{i}^{2}\right)$, for some $1 \leq i \leq n$.

The next theorem provides necessary and sufficient conditions for a Gaussian ring $R$ to satisfy $w \cdot \operatorname{dim} R \leq 1$.

Theorem 2.2. Let $R$ be a ring. Then the following conditions are equivalent:

1. $w \cdot \operatorname{dim} R \leq 1$.

2. $R$ is a Gaussian PF ring.

3. $R$ is a Gaussian reduced ring.

Proof. We show that $1 \rightarrow 2 \rightarrow 3 \rightarrow 1$ :

$1 \rightarrow 2$. If $w \cdot \operatorname{dim} R \leq 1$, then all ideals of $R$ are flat. In particular, $R$ is a PF ring. We also conclude that localizations of $R$ by prime ideals are domains of weak global dimension at most one, that is, they are valuation domains. As valuation domains are Gaussian, $R$ is locally, and therefore globally Gaussian.

$2 \rightarrow 3$. Since PF rings are locally domains, $R$ is a reduced ring.

$3 \rightarrow 1$. Since $R$ is a reduced ring every localization of $R$ by a prime ideal $R_{P}$, is a local, reduced, Gaussian ring. Let $a$ and $b$ in $R_{P}$ satisfy $a b=0$. By Lemma 2.1. $(a, b)^{2}=\left(b^{2}\right)$ or $\left(a^{2}\right)$. Say $(a, b)^{2}=\left(b^{2}\right)$, then $a^{2}=t b^{2}$ for some $t$ in $R_{P}$. Thus $a^{3}=t b(a b)=0$. Since $R_{P}$ is reduced, $a=0$, and $R_{P}$ is a domain. Therefore, $R_{P}$ is a Prüfer local domain, that is, $R_{P}$ is a valuation domain. It follows that $w \cdot \operatorname{dim} R_{P} \leq 1$ for all prime ideal $P$ of $R$, and as the supremum of all $w \cdot \operatorname{dim} R_{P}$, $w \cdot \operatorname{dim} R \leq 1$.

In particular, we note that a Gaussian ring $R$ is reduced if and only if it is locally a domain.

We now characterize when a Gaussian ring is semihereditary.

Theorem 2.3. Let $R$ be a ring. Then the following conditions are equivalent:

1. $R$ is a semihereditary ring.

2. $R$ is a Gaussian PP ring.

3. $R$ is a Gaussian ring and $Q(R)$ is a Von Neumann regular ring.

Proof. We show $1 \rightarrow 2 \rightarrow 3 \rightarrow 1$ :

$1 \rightarrow 2$. Since a semihereditary ring $R$ has $w \cdot \operatorname{dim} R \leq 1$, it is a Gaussian ring. Since all its finitely generated ideals are projective, $R$ is a PP ring.

$2 \rightarrow 3$. By [5, Theorem 4.2.10] the PP condition implies that $Q(R)$ is a Von Neumann regular ring.

$3 \rightarrow 1$. Let $\mathrm{P}$ be a prime ideal of $R$. By [5, Corollary 4.2.19], it suffices to prove that $R_{P}$ is a valuation domain. Since $Q(R)$ is Von Neumann regular it is a reduced ring. It follows that $R$ is a reduced ring. Therefore, every localization of $R$ by a 
prime ideal $R_{P}$, is a local, reduced, Gaussian ring. We now argue that $R_{P}$ is a valuation domain, as in Theorem $2.2(3 \rightarrow 1)$.

In general a ring with Von Neumann regular total ring of quotients need not be a PP ring. Example 2.5 exhibits a ring with Von Neumann regular total ring of quotient which is not even a PF ring. We note that Theorem 2.3 implies that the PP condition coincides with the Von Neumann regularity of the total ring of quotients condition if the ring is Gaussian.

As localizations of Gaussian rings are Gaussian rings, the total ring of quotients of a Gaussian ring is a Gaussian ring, but, by the above theorem it may not necessarily be Von Neumann regular (for an explicit example see Section 3, Example 3.4). This observation raises the question whether all rings which are total rings of quotients, that is, every element is either a unit or a zero divisor, are Gaussian. The following example shows that this is not the case.

Example 2.4. A non-Gaussian total ring of quotients.

Quentel ([11], and see [5, page 120] for an error-free version) constructed an example of a ring $R$ satisfying:

1. $R$ is a reduced ring.

2. $R=Q(R)$.

3. Min $R$, the set of all minimal prime ideals of $R$ in the induced Zariski topology, is compact.

4. $R$ is not a Von Neumann regular ring.

Assume that $R$ is a Gaussian ring. Since $R$ is reduced, it is locally a domain. But then $R$ is a PF ring. Since Min $R$ is compact, but $R=Q(R)$ is not Von Neumann regular, this contradicts Theorem 2.4.10 of [5].

We do not know the exact conditions under which a total ring of quotients is a Gaussian ring, but it is interesting to note that a nontrivial (that is, not a field) total ring of quotients of a ring may be Gaussian without the ring itself being Gaussian.

Example 2.5. A Gaussian total ring of quotients of a non-Gaussian ring.

Let $R$ be a Noetherian, local, reduced ring, which is not a domain. Clearly such a ring cannot be Gaussian. Since $R$ is Noetherian, Min $R=\left\{P_{1}, \ldots, P_{n}\right\}$ is a finite set. The total ring of quotients of $R, Q(R)=R_{P_{1}} \oplus \ldots \oplus R_{P_{n}}$. Each $R_{P_{i}}$ is a field, therefore Gaussian; and as a direct sum of Gaussian rings, $Q(R)$ is a Gaussian ring.

\section{Coherent Gaussian Rings}

Recall that the small finitistic projective dimension of a ring $R$, denoted by $f P \cdot \operatorname{dim} R$, is equal to the supremum of the projective dimensions of $R$ modules $M$, which satisfy proj.dim $\operatorname{dim}_{R}<\infty$, and $M$ admits a finite resolution consisting of finitely generated projective modules. In general, $f P \cdot \operatorname{dim} R \leq w \operatorname{dim} R$. When $R$ is a coherent ring, $f P$.dim $R$ accept a more manageable form, namely:

$f P . \operatorname{dim} R=\sup \left\{\right.$ proj. $\operatorname{dim}_{R} M \mid M$ is finitely presented and $\left.\operatorname{proj} \operatorname{dim}_{R} M<\infty\right\}$.

Recall that a ring $R$ is called a regular ring if every finitely generated ideal of $R$ has finite projective dimension. This notion, extending Noetherian regularity, was extensively studied for coherent rings. Coherent rings of finite weak global 
dimensions are regular rings. In particular, Von Neumann regular rings and semihereditary rings are regular rings. But, there are coherent rings, even local, with infinite weak global dimension which are regular.

Lemma 3.1. Let $R$ be a local, coherent, regular ring, then $f P . \operatorname{dim} R=w \cdot \operatorname{dim} R$.

Proof. Since $R$ is a coherent ring, any finitely presented module satisfies $w \cdot \operatorname{dim}_{R} M$ $=$ proj. $\operatorname{dim}_{R} M$. Such a module admits a resolution of finitely generated free modules. As $R$ is a coherent regular ring, any finitely generated ideal of $R$ has finite projective dimension, hence any finitely presented cyclic $R$ module has finite projective dimension. It follows by induction on the number of generators

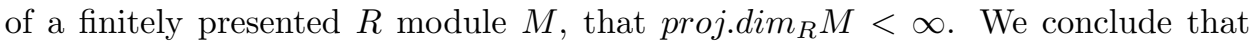
$f P \cdot \operatorname{dim} R=w \cdot \operatorname{dim} R$.

The close relation between the small finitistic projective dimension and the weak global dimension, makes it natural to involve $f P \cdot \operatorname{dim} R$, in any attempt to track the possible values of $w \cdot \operatorname{dim} R$, for a Gaussian ring $R$. In case $R$ is a coherent Gaussian ring, Theorem 3.2 shows that $f P \cdot \operatorname{dim} R \leq 1$. Moreover, the finitistic projective dimension of such a ring seems to capture "the finite part" of its weak global dimension, that is, as we see in Theorem 3.3, the weak global dimension is either locally equal to the finitistic projective dimension, and therefore globally less or equal to 1 , or it is infinite.

Theorem 3.2. Let $R$ be a coherent Gaussian ring, then $f P . \operatorname{dim} R \leq 1$.

Proof. Assume first that $R$ is local with maximal ideal $m$. We consider two cases:

Case 1. All the elements of $m$ are zero divisors.

We will show that in this case $f P . \operatorname{dim} R=0$. Let $I=\left(a_{1}, \ldots, a_{n}\right)$ be a finitely generated proper ideal of $R$. By Lemma 2.1., $I^{2}=\left(a_{i}^{2}\right)$ for some $1 \leq i \leq n$. Let $c \neq 0$ satisfy $c a_{i}^{2}=0$, then $c I^{2}=(c I) I=0$. It follows that either $c I=0$, or, if not, there exists an element $b \in I$ such that $c b \neq 0$. But, in this second case we obtain $c b I \subseteq(c I) I=0$. We conclude that $I$ has a nonzero annihilator. According to [5], Corollary 3.3.17], fP.dim $R=0$. Note that the coherence of $R$ was not necessary for this case.

Case 2. $m$ contains a regular element $a \epsilon m$.

We will show that in this case $f P \cdot \operatorname{dim} R=1$. Since $R$ is a coherent ring, by [5], Corollary 3.1.4] we have $f P . \operatorname{dim} R=f P . \operatorname{dim} R / a R+1$. Thus it suffices to show that $f P \cdot \operatorname{dim} R / a R=0$. Now, as a homomorphic image of a Gaussian ring, $R / a R$ is a Gaussian ring. $R / a R$ is local with maximal ideal $m / a m$, and by Case 1 , it suffices to show that $m / a m$ consists entirely of zero divisors. Denote $R / a R$ by $\bar{R}$, and elements of $\bar{R}$ by $\bar{r}$. Let $\overline{0} \neq \bar{b} \epsilon \bar{m}$. We need an element $\overline{0} \neq \bar{c}$ in $\bar{R}$ such that $\overline{b c}=\overline{0}$ in $\bar{R}$. By Lemma $2.1(a, b)^{2}=\left(a^{2}\right)$ or $\left(b^{2}\right)$.

If $(a, b)^{2}=\left(a^{2}\right)$, then $b^{2} \epsilon\left(a^{2}\right) \subset(a)$, and we may take $c=b$.

If $(a, b)^{2}=\left(b^{2}\right)$, then $a^{2} \epsilon\left(b^{2}\right)$. We therefore have a $t \epsilon R$ satisfying $a^{2}=t b^{2}$. It follows that $\overline{t b^{2}}=\overline{0}$ in $\bar{R}$. If $\bar{t} \neq \overline{0}$, we are done, as either $\overline{t b}=\overline{0}$, or if $\overline{t b} \neq \overline{0}$, then $\overline{(t b) b}=\overline{0}$.

If $\bar{t}=\overline{0}$, then $t=a r$, for some $r \epsilon R$. We then have $a^{2}=t b^{2}=a r b^{2}$. Since $a$ is a nonzero divisor $a=r b^{2}$.

If $\bar{r} \neq \overline{0}$, we are done as either $\overline{r b}=\overline{0}$ or if $\overline{r b} \neq \overline{0}$, then $\overline{(r b) b}=\overline{0}$. 
If $\bar{r}=\overline{0}$, then $r=a s$ for some $s \in R$. We then have $a=r b^{2}=a s b^{2}$. Since $a$ is a nonzero divisor $1=s b^{2}$, contradicting the fact that $b$ is not a unit. Thus $f P . \operatorname{dim} R=1$.

Assume now that $R$ is not local. Let $M$ be a finitely presented $R$ module with proj. $\operatorname{dim}_{R} M<\infty$. Then for a maximal ideal $m$ of $R$,

$$
\operatorname{proj.dim}_{R_{m}} M_{m} \leq \text { proj.dim } \operatorname{dim}_{R} M<\infty \text {. }
$$

It follows that

$$
\text { proj.dim } R_{m} M_{m} \leq f P . \operatorname{dim} R_{m} \leq 1 .
$$

Since over coherent rings the projective and weak dimensions of a finitely presented module coincide, we obtain

$$
\begin{aligned}
\text { proj.dim }_{R} M & =\text { w.dim } \\
& =\sup \left\{w \cdot \operatorname{dim}_{R_{m}} M_{m} \mid m \text { runs over the maximal ideals of } R\right\} \\
& =\sup \{\text { proj.dim } \\
& \leq 1 .
\end{aligned}
$$

We conclude that $f P \cdot \operatorname{dim} R \leq 1$ as desired.

Finitely generated ideals of Von Neumann regular rings are projective, hence a Von Neumann regular ring is semihereditary. Nevertheless, it is customary to call semihereditary rings $R$ of $w$. $\operatorname{dim} R=0$ Von Neumann regular, and semihereditary rings $R$ of $w \cdot \operatorname{dim} R=1$ semihereditary.

Theorem 3.3. Let $R$ be a coherent Gaussian ring. Then either $w \cdot \operatorname{dim} R \leq 1$, or $w \cdot \operatorname{dim} R=\infty$. In particular, if $R$ is a regular ring, then $R$ is either a Von Neumann regular ring or a semihereditary ring.

Proof. Assume that $R$ is a regular ring. By Lemma 3.1 at every localization by a maximal ideal $m$, we have $w \cdot \operatorname{dim} R_{m}=f P \cdot \operatorname{dim} R_{m} \leq 1$. We conclude that

$$
w \cdot \operatorname{dim} R=\sup \left\{w \cdot \operatorname{dim} R_{m} \mid m \text { runs over all maximal ideals of } R\right\} \leq 1 .
$$

If $w \cdot \operatorname{dim} R=0, R$ is a Von Neumann regular ring. If $w \cdot \operatorname{dim} R=1$, since $R$ is coherent it is a semihereditary ring. We finish by noting that nonregular coherent rings have infinite weak dimension.

We conclude this article with two examples that show that both values of the $f P \cdot \operatorname{dim} R, 0$ and 1 , are realizable when $w \cdot \operatorname{dim} R=\infty$.

Example 3.4. A local, Noetherian, Gaussian $\operatorname{ring} R$ with $w \cdot \operatorname{dim} R=\infty$, and $f P . \operatorname{dim} R=0$.

Let $k$ be a field, and let $T$ and $U$ be indeterminates over $k$. Denote by $t$ and $u$ the images of $T$ and $U$ in $k[T, U] /(T, U)^{2}$. Let

$$
R=k[t, u]_{(t, u)} .
$$

The maximal ideal $m=(t, u)$ satisfies $m^{2}=0$, hence $R$ is Gaussian. If $R$ is a regular ring by Theorem 3.3, it is either Von Neumann regular or semihereditary. Since $\mathrm{R}$ is local, this means it must be a domain. We conclude that $w \cdot \operatorname{dim} R=\infty$. We remark that it is possible to show directly that proj.dim $R_{m}=\infty$. Clearly every proper finitely generated ideal of $R$ admits a nonzero annihilator, $(t, u)$, therefore $f P . \operatorname{dim} R=0$. 
Example 3.5. A local, Noetherian, Gaussian ring $R$ with $w \cdot \operatorname{dim} R=\infty$, and $f P . \operatorname{dim} R=1$.

Let $C$ be the complex numbers, and let $X$ and $Y$ be indeterminates over $C$. Denote by $x$ and $y$ the images of $X$ and $Y$ in

$$
R=C[X, Y] /\left(Y X+Y, Y^{2}\right)=C[x, y] .
$$

It is shown in [1] that $R$ is a Gaussian ring of Krull dimension one, with unique minimal prime ideal $P=(y)$, and all maximal ideals, but $M=(x+1, y)$, invertible. $R$ is not regular. One way to see this is to note that $R_{M}$ is not regular since it is not a domain, as $y(x+1)=0$. In particular, w.dim $R_{M}=\infty$, which implies $w \cdot \operatorname{dim} R=\infty$. According to Theorem $3.2 f P \cdot \operatorname{dim} R \leq 1$. To show equality it suffices to find a finitely presented $R$ module $M$, with $\operatorname{proj} \operatorname{dim}_{R} M=1$. For that purpose it suffices to find one prime ideal of $R, Q$, such that proj.dim $R_{Q} M_{Q}=1$. Let $Q$ be an invertible maximal ideal of $R$. $Q R_{Q}$ is principal and therefore $R_{Q}$ is a DVR. Thus $R_{Q}$ is a coherent regular ring, and by Lemma 3.1, fP.dim $R_{Q}=w \cdot \operatorname{dim} R_{Q}=1$. Thus the existence of such a module $M_{Q}$ is guaranteed. One can also find such a module by a direct computation, for example, $M_{Q}=R_{Q} / Q R_{Q}$.

\section{REFERENCES}

[1] D.D. Anderson, Another generalization of principal ideal rings, J. Algebra 48 (1997), 409416. MR0453728 (56:11988)

[2] D.D. Anderson and V. Camillo, Armendariz rings and Gaussian rings, Comm. Algebra 26 (1998), 2265-2272. MR 1626606 (99e:16041)

[3] A. Corso and S. Glaz, Gaussian ideals and the Dedekind-Mertens Lemma, Marcel Dekker, Lecture Notes Pure Appl. Math 217 (2001), 131-143. MR1824223 (2002b:13015)

[4] R. Gilmer, Multiplicative ideal theory, Marcel Dekker, 1972. MR0427289 (55:323)

[5] S. Glaz, Commutative coherent rings, Springer-Verlag, Lecture Notes no. 1371, 1989. MR0999133 (90f:13001)

[6] S. Glaz and W.V. Vasconcelos, Gaussian polynomials, Marcel Dekker, Lecture Notes 186 (1997), 325-337. MR1422491 (97m:13012)

[7] S. Glaz and W.V. Vasconcelos, The content of Gaussian polynomials, J. Algebra 202 (1998), 1-9. MF1614237 (99c:13003)

[8] W. Heinzer and C. Huneke, Gaussian polynomials and content ideals, Proc. Amer. Math. Soc. 125 (1997), 739-745. MR 1401742 (97e:13015)

[9] K. A. Loper and M. Roitman, The content of a Gaussian polynomial is invertible, preprint.

[10] T. Lucas, Gaussian polynomials and invertibility, preprint.

[11] Y. Quentel, Sur la compacité du spectre minimal d'un anneau, Bull. Soc. Math. France 99 (1971), 265-272. MR0289496 (44:6685)

[12] H. Tsang, Gauss's Lemma, Ph.D. Thesis, University of Chicago, 1965.

Department of Mathematics, University of Connecticut, Storrs, Connecticut 06269

E-mail address: glaz@uconnvm.uconn.edu 